\title{
Epidemiology of subsequent bloodstream infections in the ICU
}

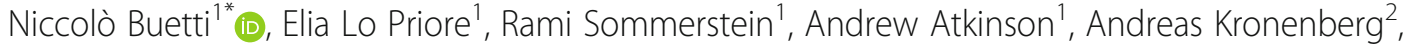 \\ Jonas Marschall ${ }^{1}$ and the Swiss Centre for Antibiotic resistance (ANRESIS)
}

\begin{abstract}
Subsequent bloodstream infections (sBSI) occur with a delay after removal of the intravascular catheter (IVC) whose tip revealed microbial growth. Here we describe the epidemiology of sBSI in the intensive care setting. Serratia marcescens, Staphylococcus aureus, Pseudomonas aeruginosa, and yeast were the pathogens most frequently associated with sBSI. In contrast, Enterococci were rarely found in sBSI.
\end{abstract}

\section{Letter}

A recently published review on the management of catheter-related infection highlighted the clinical importance of a positive catheter culture without concomitant positive blood cultures in the ICU [1]. Recently, we conducted a nationwide, observational study on all positive intravascular catheter (IVC) tip cultures in Switzerland investigating subsequent bloodstream infections (i.e., bloodstream infection occurring after the catheter has been removed) with non-ICU and ICU data [2]. Interestingly, the studies investigating this topic reported either data from an individual hospital [1] or focused on single pathogens $[3,4]$. Moreover, only one observational study studied the ICU population [5]. Based on the Swiss Antibiotic Resistance Surveillance System (ANRESIS), we aimed to describe the current epidemiology of culturepositive IVC tips without concurrent bacteremia in the ICU and to characterize bacteremia or fungemia occurring after catheter removal.

We conducted a nationwide surveillance study on all positive IVC tip cultures recovered in Swiss ICUs (36 hospitals) from 2008 to 2015. An IVC tip culture, which required IVC removal, was included in the analysis if at least one microorganism could be cultivated. We excluded data from patients with concurrent bacteremia and fungemia with the same microorganism identified 7 days before to 2 days after IVC removal (623 cases). Subsequent bloodstream infection (sBSI) was defined as isolating (from blood cultures performed $>2$ days up to 7 days after IVC removal) the same microorganism as the one recovered from the IVC tip.

Over the 8-year period, 2,941 positive IVC tip cultures without concurrent bacteremia were identified in ICUs. In 3.1\% (92/2,941, 95\% confidence interval 2.5-3.8) of removed catheters an sBSI was observed (Fig. 1). Among bacterial microorganisms, Serratia marcescens (4/40, 10\%, 3.3-24), Staphylococcus aureus $(7 / 88,8.0 \%, 3.5-16.2)$ and Pseudomonas aeruginosa $(4 / 81,4.9 \%, 1.6-12.8)$ were the most frequently identified agents causing sBSI. Subsequent fungemia developed in $8 / 29(27.6 \%, 11.3-43.9)$ IVC tips positive for fungi (Additional file 1: Table S1). Enterococci rarely caused sBSI $(1.6 \%, 0.5-4.2)$.

To our knowledge, ours is the largest epidemiologic description of sBSI in this setting. Our findings highlight that particular attention should be paid if Candida albicans, S. aureus, S. marcescens, and P. aeruginosa are detected on an IVC tip. The presence of these four microorganisms is associated with a higher frequency of sBSI than other microorganisms and, therefore, a short treatment may need to be considered by intensive care physicians. In contrast, enterococci represented the lowest risk for sBSI and probably do not require specific antimicrobial therapy.

\footnotetext{
* Correspondence: niccolo.buetti@gmail.com

1 Department of Infectious Diseases, University Hospital Bern, Bern,

Switzerland

Full list of author information is available at the end of the article
} 


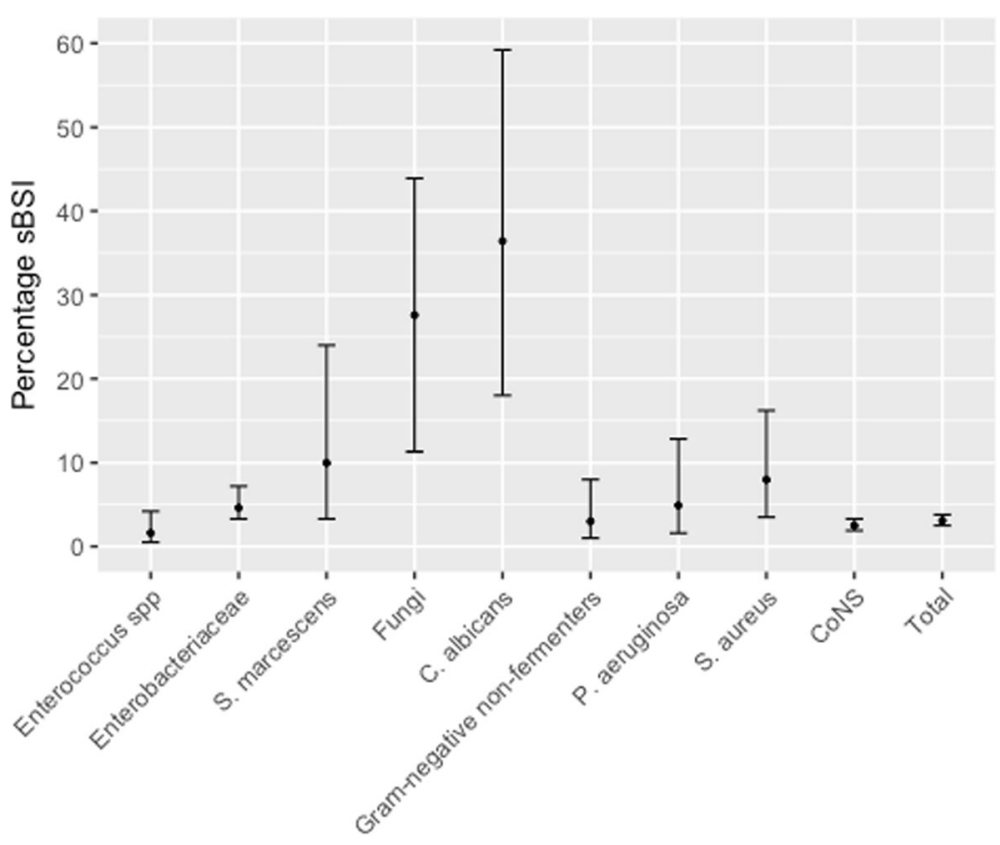

Microorganism

Fig. 1 Proportion and confidence intervals of subsequent bloodstream infections (sBS) in the ICU

\section{Additional file}

Additional file 1: Table S1. Microorganism distribution of positive catheter tip culture and sBSI in the ICU. (DOCX $16 \mathrm{~kb}$ )

\section{Acknowledgments}

We thank all microbiology laboratories participating in the ANRESIS network: Institute for Laboratory Medicine, Cantonal Hospital Aarau; Central Laboratory, Microbiology Section, Cantonal Hospital Baden; Clinical Microbiology, University Hospital Basel; Viollier AG, Basel; Laboratory Medicine EOLAB, Department of Microbiology, Bellinzona; Institute for Infectious Diseases, University Bern; Microbiology Laboratory, Unilabs, Coppet; Central Laboratory, Cantonal Hospital Graubünden; Microbiology Laboratory, Hospital Thurgau; Microbiology Laboratory Hôpital Fribourgeois, Fribourg: Bacteriology Laboratory, Geneva University Hospitals, Geneva: ADMED Microbiology, La Chaux-de-Fonds; Institute for Microbiology, Université de Lausanne; Centre for Laboratory Medicine, Cantonal Hospital Luzern; Centre for Laboratory Medicine, Cantonal Hospital Schaffhausen; Centre for Laboratory Medicine Dr. Risch, Schaan; Central Institute, Hôpitaux Valaisans (ICHV), Sitten; Centre of Laboratory Medicine St. Gallen; Institute for Medical Microbiology, University Hospital Zürich; Laboratory for Infectious Diseases, University Children's Hospital Zürich.

We would also like to thank the steering committee of ANRESIS. ANRESIS collaboration group: A. Burnens, Synlab Suisse, Switzerland; A.

Cherkaoui, Bacteriology Laboratory, Geneva University Hospitals, Switzerland; V. Gaia, Department of Microbiology, EOLAB, Bellinzona, Switzerland; O. Dubuis, Viollier AG, Basel, Switzerland; A. Egli, Clinical Microbiology Laboratory, University Hospital Basel, Switzerland; D. Koch, Federal Office of Public Health, Bern, Switzerland; A. Kronenberg, Institute for Infectious Diseases, University of Bern, Switzerland; P. Nordmann, Molecular and Medical Microbiology, Department of Medicine, University Fribourg, Switzerland; V. Perreten, Institute of Veterinary Bacteriology, University of Bern, Switzerland; J.-C. Piffaretti, Interlifescience, Massagno, Switzerland; G Prod'hom, Institute of Microbiology, Centre Hospitalier Universitaire Vaudois, Lausanne, Switzerland; J. Schrenzel, Bacteriology Laboratory, Geneva University Hospitals, Geneva, Switzerland; S. L. Leib, Institute for Infectious Diseases, University of Bern, Switzerland; A. F. Widmer, Division of Infectious Diseases \& Hospital Epidemiology, University of Basel, Switzerland; G. Zanetti,
Service of Hospital Preventive Medicine, Centre Hospitalier Universitaire Vaudois, Lausanne, Switzerland; R. Zbinden, Institute of Medical Microbiology, University of Zürich, Switzerland.

\section{Funding}

The ANRESIS database is funded by the Federal Office of Public Health, and the University of Bern, Switzerland.

\section{Availability of data and materials}

The datasets generated and/or analyzed during the current study are not publicly available but are available from the corresponding author on reasonable request.

\section{Authors' contributions}

$N B, R S, J M$, and ELP conceived and designed the study. NB, AA, and RS analyzed the data. NB, RS, JM, AK, and ELP wrote the manuscript. All authors contributed to the discussion and reviewed the manuscript. The ANRESIS program provides antibiotic resistance data for all routinely collected microbiological samples from 20 Swiss laboratories (see Acknowledgments), each of them collecting microbiological data from different acute care hospitals distributed across the country. All authors commented and approved the final version of the paper.

\section{Ethics approval and consent to participate}

As the analysis was performed on anonymized non-genetic surveillance data ethical consent was not required according to the Swiss law for research on humans (Art. 33 al. 2 LRH)

\section{Consent for publication}

Not applicable.

\section{Competing interests}

The authors declare that they have no competing interests.

\section{Publisher's Note}

Springer Nature remains neutral with regard to jurisdictional claims in published maps and institutional affiliations. 


\section{Author details}

'Department of Infectious Diseases, University Hospital Bern, Bern,

Switzerland. ${ }^{2}$ Institute for Infectious Diseases, University of Bern, Bern,

Switzerland.

Received: 7 July 2018 Accepted: 23 July 2018

Published online: 11 October 2018

\section{References}

1. Timsit JF, Rupp M, Bouza E, et al. A state of the art review on optimal practices to prevent, recognize, and manage complications associated with intravascular devices in the critically ill. Intensive Care Med. 2018;44:742-759

2. Buetti N, Lo Priore E, Atkinson A, Swiss Centre for Antibiotic R, et al. Low incidence of subsequent bacteraemia or fungaemia after removal of a colonized intravascular catheter tip. Clin Microbiol Infect. 2018;24:548.e1-3.

3. Perez-Parra A, Munoz P, Guinea J, et al. Is candida colonization of central vascular catheters in non-candidemic, non-neutropenic patients an indication for antifungals? Intensive Care Med. 2009;35:707-12.

4. Hetem DJ, de Ruiter SC, Buiting AG, et al. Preventing staphylococcus aureus bacteremia and sepsis in patients with staphylococcus aureus colonization of intravascular catheters: a retrospective multicenter study and metaanalysis. Medicine. 2011;90:284-8.

5. Mrozek N, Lautrette A, Aumeran C, et al. Bloodstream infection after positive catheter cultures: what are the risks in the intensive care unit when catheters are routinely cultured on removal? Crit Care Med. 2011;39:1301-5. 